\title{
Applying Self-Organizing Maps Method to Analyze the Corrective Action's Quality Provided to Customers with Mobile Terminals
}

\author{
Andi Mwegerano ${ }^{1}$, Pekka Kytösaho ${ }^{1}$, Aulis Tuominen ${ }^{2}$ \\ ${ }^{1}$ Nokia Corporation, Salo, Finland; ${ }^{2}$ University of Turku Ylhäistentie, Salo, Finland. \\ Email: andi.mwegerano@nokia.com
}

Received March 29 ${ }^{\text {th }}, 2012$; revised April 9 ${ }^{\text {th }}, 2012$; accepted April 30 ${ }^{\text {th }}, 2012$

\begin{abstract}
In the after market phase, responding to issues raised by customers within a reasonable time is crucial. Another factor which is important to customers is the quality of the issue corrective actions (QoiCA). This paper analyses the QoiCA from the user's perspective, regarding the quality of corrective actions provided through an in-built tool (GENIUS) used within corporations. A survey questionnaire was sent to different participants in the network chain which handles or resolves the issues. The participants were from 17 European countries and 7 non-European countries. Responses were analysed, using statistical methods and the Self Organising Map (SOM) model and results were used to pinpoint or suggest the areas that are seen as opportunities for improving the quality of the corrective actions provided. Higher quality of corrective actions, along with other initiatives, will help to improve customer's satisfaction. Three of the clear issues observed in this paper that contribute to long issue resolution time (iRT) are: 1) Long time to receive samples; 2) High frequency for asking more information from lower levels; and 3) Business impact price tag, to allow the issues to be prioritized, were missing from the escalated issues. QoiCA is jeopardized when: 1) A poor description of the issue is provided by the creator of the issue; 2) A poor response to the default requested additional questions (information) regarding the reported issue on top of the issue description. The authorized service vendors (ASV) users need more training with the in-built tool so that they know, for example, where to get help when they need it. When customer issues are resolved satisfactorily, there is a much higher chance that the customers involved will remain satisfied and loyal.
\end{abstract}

Keywords: Self-Organizing Map (SOM); Customer Satisfaction; Corrective Actions; Issue; Issue Description

\section{Introduction}

Customers who do complain give a firm the chance to correct issues (including some the firm may not even know it has), restore relationships with the customer, and improve future satisfaction for all. Service recovery efforts play a crucial role in achieving (or restoring) customer satisfaction. When complaints are resolved satisfactorily, there is a much higher chance that the customers involved will remain loyal. Issues presented by customers should be seen as a profit center not a cost center. When a dissatisfied customer defects, the firm loses more than just the value of the next transaction. It may also lose a long-term stream of profits from the customer, and from anyone else who switches suppliers or is deterred from doing business with that firm because of negative comments from unhappy friends [1]. TARP research found that intentions to repurchase for different types of products ranged from 9\% to 37\% when customers were dissatisfied but did not complain. For the majority of customer issues, the retention rate increased from $9 \%$ to $19 \%$ if the customer issue presented to a firm offered a sympathetic ear but was unable to resolve the issue to the satisfaction of the customer. If the issue could be resolved to the satisfaction of the customer the retention rate jumped to $54 \%$. The highest retention rate, $82 \%$ was achieved when issues were fixed quickly-typically on the spot [2].

\section{Customer Satisfaction}

It is not enough to fulfill the basic needs and expectations of the customers. In order to create loyal and satisfied customers the firms have to delight their customers continuously and also aim to exceed customers' expectations.

Noraki Kano has developed a model for customer satisfaction, where quality dimensions are divided in three groups: basic needs, expected needs and exciting experiences. The basic needs are almost unconsciously ex- 
pected to be there by the customer. Expected needs are such needs as the customer is aware of and wants to have satisfied but they are not always absolutely necessary. The exciting experiences, however, are items the manufacturer must find by themselves. These are surprises to the customer, who sometimes cannot imagine them. Surprises need not be limited to products or product features but may also be services. The degree of customer satisfaction depends on the correlation between customer expectation and his/her experience, but it is also influenced by such things as the image of the company [3].

Sometimes it is possible to change dissatisfaction to excitement. By treating a disappointed customer very well a firm can win a loyal customer. Here it is important that people in the front line have sufficient knowledge and the possibility to act rapidly and take corrective actions when an issue occurs [4]. This study work has attempted to analyze different activities in a service chain network, which contribute to the quality of QoiCA and the iRT taken to provide CA. This paper is organized as follows: firstly we describe the data used and its acquisition and process for customer issues escalation in Section 2 to 2.2. In Section 3 to 3.4 we explain the methodology and basic theory of the self-Organizing Maps and why we choose this method for analyzing the survey data we collected. In Section 4 to 4.3 are the results and findings of the dependent variable data; the results are tabulated and explained. In Section 5 to 5.5 are results of the independent variables which are used to clarify some issues that need attention for improvement in the dependent variables discussed in Section 4 to 4.3. Finally, in Section 6 , the findings are summarized and recommendations from the survey and further research questions are outlined.

\section{Data Description and Acquisition}

In order to analyze the time and the quality of corrective actions provided to the customers, a questionnaire was sent to different levels of users of the inbuilt-tool located in 17 different European countries and 7 non-European countries. A set of 36 survey questions was sent to different level users of the in-built tool (GENIUS) used for reporting and resolving issues raised by customers. The questions were segmented into two main groups namely independent variables and dependent variables. The dependent variables include: The survey on quality and time spent in resolving issues raised by customers; and the quality and quantity of issue description. The independent variables include the following: Usage of the tool, training, support, different product categories and working experience with Nokia products. Figure 1 displays the information structure about different countries and services levels in GENIUS tool participated in the survey.

The last digit in the diagram represents the level of the service in the chain network i.e., L1, L2, L3, L4. The rest of digit(s) in the diagram represent different countries in Europe as well as somewhere else where the participants were located during this study. For example for number 152, 15 is the coded number for a country and service level is $\mathrm{L} 2$.

Figure 2 shows the total number of participants in the survey from each service level.

\subsection{Color Code and Data Graphs}

Figure 3 illustrates the colour coding for score in the survey. The colour code changes and data graphs are used to discover contrast areas i.e., colour changes from

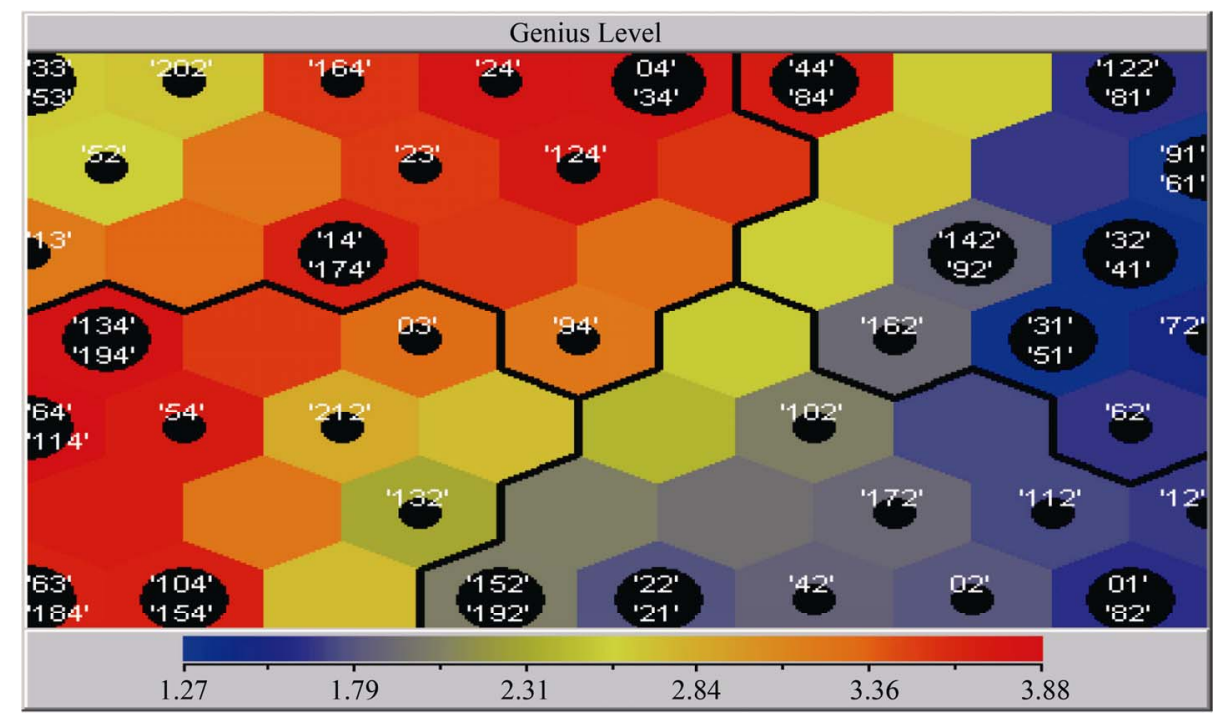

Figure 1. Different countries and level users (L1, L2, L3, and L4) in GENIUS coded in the survey results. 


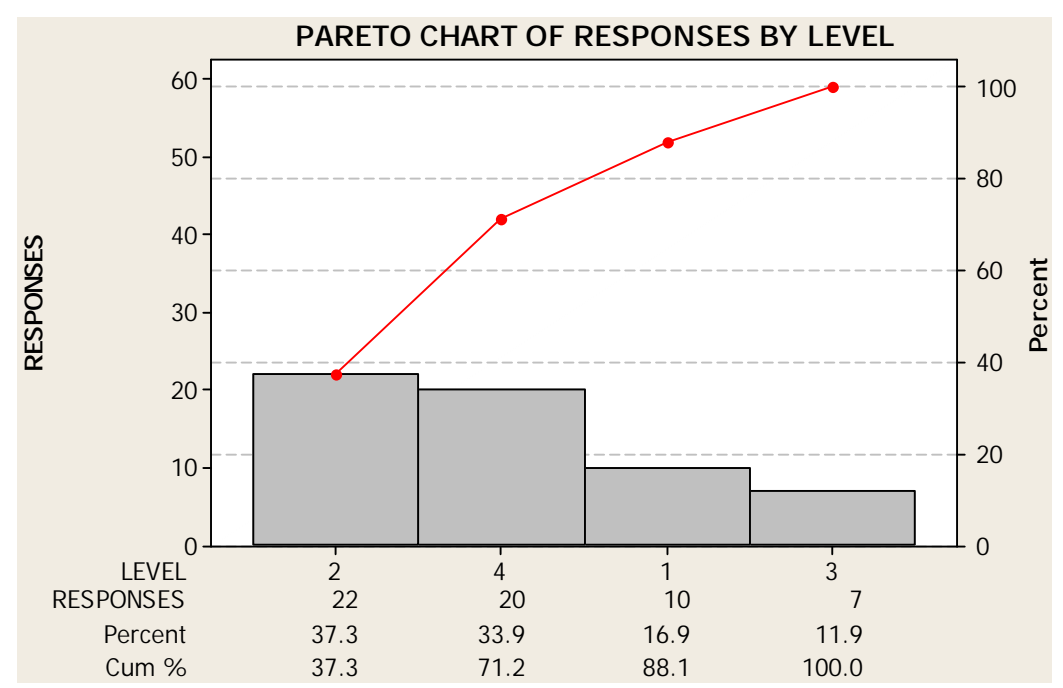

Figure 2. Pareto chart of all participants in different levels.

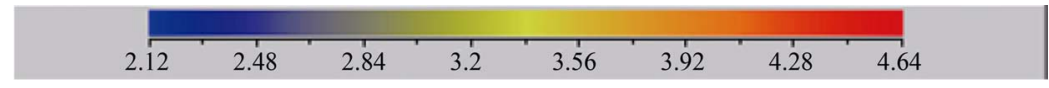

Figure 3. Colour coding for score in the survey.

blue left (e.g. score 2.12, etc.) which means excellent to red which means poor results respectively. The reddish areas, e.g. a score of 4.64 in the right end of the bar diagram, in the diagram are the opportunity in the process for improvement. The survey questions analyzed in this paper are embedded into the graph on the top of the figures and the responses are seen according to the colour contrasts in the graphs.

\subsection{Issue Escalation Process}

Figure 4 illustrates the chain network process for customer issues escalation. For example when a customer $X$ confronts an issue $\mathrm{Y}$, e.g. ringing tone of the terminal is not functioning well, he/she will contact any authorized service vendor (ASV) i.e. L1 for resolving the issue. If L1 is not able to resolve the issues, it will be escalated to a higher level of service, in this case L2. The procedure will continue in the network chain until a corrective action(s) is provided to the customer.

\section{Methodology}

Due to the nature of the data, clustering with the selforganizing map approach was found to be the most applicable method for analyzing the survey data. Analysis is done with eSOM software. Neural network, MinTab and e-SOM2 data-mining tool have been used to analyze the response from different users at various levels.

\subsection{Basic Theory of the Self-Organizing Maps}

The self-organizing Maps (SOM) is a two layer neural

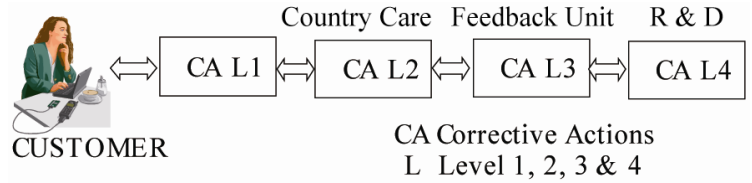

Where: CA-Corrective Actions; L1-Authorized Service Vendors (ASV); L2 - Technical Support in sales area; L3-Technical Support in region sales area; L4-Technical support at R\&D level.

Figure 4. A simplified issues escalation path diagram [6].

network that maps multidimensional data onto two dimensional topological grids [5]. The data is grouped according to similarities and patterns found, typically using the Euclidean distance as a distance measure. The results are displayed as a map of nodes, which can be divided into clusters. The SOM is allowed to freely organize itself, based on the patterns found in data, which makes the SOM a good tool for exploratory data analysis. Exploratory data analysis methods like SOM are general purpose instruments that illustrate the essential features of a data set, such as its clustering structure and relationship between its data [5].

\subsection{Similarities between Human and the Artificial Intelligence Neural Network SOM}

The human brain is dominated by the cerebral cortex, a very complex structure of neurons and hundreds of billions of synapses. The cortex includes areas that are responsible for different human activities which are associated with different sensory inputs. Each sensory input is mapped into a corresponding area of the cerebral cortex. 
The cortex is a self-organizing computational map in the human brain. The basic idea behind the Kohonen network is to set up a structure of interconnected processing units ("neurons") which compete for the signal. While the structure of the map may be quite arbitrary, most implementations support only rectangular and linear maps. The SOM defines a mapping from the input data space spanned by input vectors $X 1, X 2, X \mathrm{n}$ onto one- or two dimensional arrays of nodes. The mapping is performed in such a way that topological relationship in the n-dimensional input space is maintained when mapped to the SOM. In addition, the local density of data is also reflected by the map: areas of the input data space which are represented by more data are mapped into large area of SOM. Each node of the map is defined by a vector $W_{i j}$ which is adjusted during the training [6,9]. The Kohonen model provides a topological mapping, see Figure 5 below. The Kohonen model places a fixed number of inputs $(X)$ patterns from input layer into higher dimensional output.

\subsection{Architecture of the Kohonen Network}

The lateral connections are used to create a competition between neurons (refer Figure 6). The neuron with the largest activation level among all neurons in the output layer becomes the winner and it is the only neuron that produces an output signal. All other neuron activity is suppressed in the competition. The lateral feedback con-

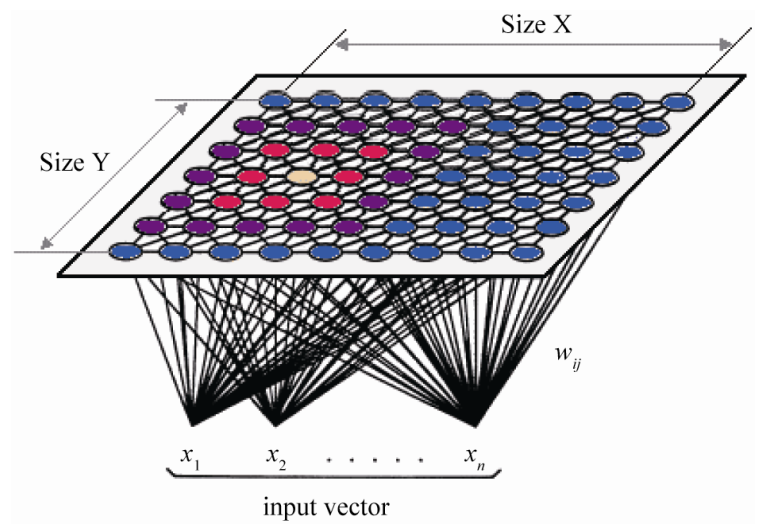

Figure 5. Feature-mapping Kohonen model [5,8].

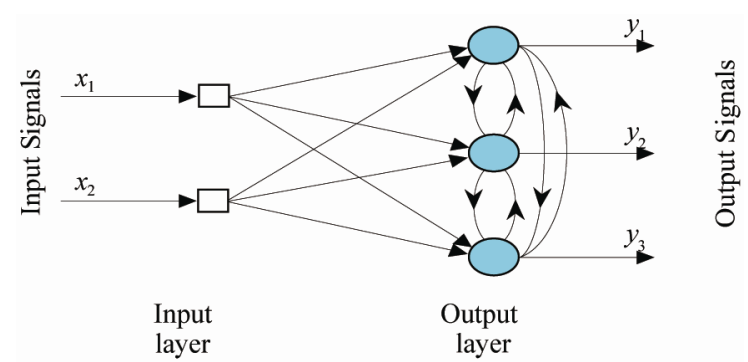

Figure 6. Architecture of the Kohonen network [5,8]. nections produce excitatory or inhibitory effects, depending on the distance from the winning neuron. This is achieved by the use of a Mexican hat function, Figure 7, which descrybes synaptic weights between neurons in the SOM layer.

\subsection{Competitive Learning Rule in SOM Network}

In SOM network, a neuron learns by shifting its weights from inactive connections to active ones. Only the winning neuron and its neighborhoods are allowed to learn. The competitive learning rule defines the change $\Delta W_{i j}$ applied to synaptic weight $W_{i j}$ as

$$
\Delta w_{i j}= \begin{cases}\alpha\left(x_{i}-w_{i j}\right), & \text { if neuron } j \text { wins the race } \\ 0, & \text { if neuron } j \text { loses the race }\end{cases}
$$

Equation (1) [5,8]

where $X_{i}$ is the input signal and $\alpha$ is the learning rate parameter. the learning parameter lies in the range between 0 and 1.

The overall effect of the competitive learning rule resides in moving the synaptic weight $W_{j}$ of the winning neuron $\mathrm{j}$ towards the input pattern $X$.

The matching criterion is equivalent to the minimum Euclidean distance between vectors. The Euclidean distance between a pair of n-by- 1 vectors $X$ and $W_{j}$ is defined by the following equation.

$$
d=\left\|\mathbf{X}-\mathbf{W}_{j}\right\|=\left[\sum_{i=1}^{n}\left(x_{i}-w_{i j}\right)^{2}\right]^{1 / 2} \quad \text { Equation (2) }[7,8]
$$

where $x_{i}$ and $w_{i j}$ are the ith elements of the vectors $X$ and $W_{j}$, respectively.

To identify the winning neuron from the competition, $j_{x}$ that best matches the input vector $X$, the following condition is applied:

$$
j_{X}=\min _{j}\left\|X-W_{j}\right\|, \quad \text { Equation (3) [5] }
$$

$j=1,2, \ldots, m$. Where $m$ is the number of neurons in the Kohonen layer.

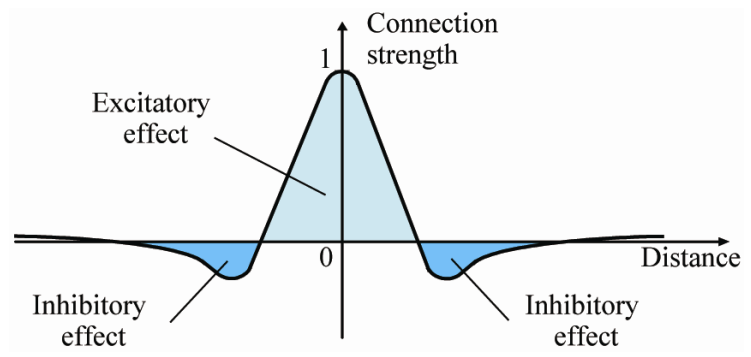

Figure 7. The Mexican hat function of lateral connection [5, 8]. 


\section{Results and Findings}

The map training phase is based on the GENIUS level of users and all the responses. The dataset is non-weighted and normalized. Original data values are in the graphs. All the graphs included are from the same model, and data points are placed in the same position in all graphs. The color code changes and data graphs are used to discover contrast areas.

\subsection{Dependent Variables (Response)}

Eight different questions see Table 1. Below, form the dependent variables which are used to evaluate the quality and iRT for providing CA. More dependent variables, description and additional questions are included to maximize the quality and iRT of the corrective actions provided in resolving issues raised by customers.

Table 1 shows that the quality of corrective actions from lower levels is associated well with iRT and descriptions of the issues raised by end customers, while the quality of additional questions correlates to the quantity of additional questions.
Figure 8, enhances the results tabulated in Table 1, i.e. the figure tells about two principle components i.e. data structure of the process. The first and second components are independent data variations. From the principal component analysis figure it can be observed that, the responses of Q3, Q5 and Q4 are closely correlated and that the response of Q7 are loosely correlated to the response of Q1 and Q8.

From Figure 9, it can be concluded that on average, most of the respondents ranked the corrective actions provided from upper level as either good or satisfactory. Few were in the extreme ends of either excellent or poor. Also it can be noted that the L2 users are quite satisfied with responses they get from the lower level, while some L4 are extremely unsatisfied with the response they get from lower levels.

From Figure 10, it can be observed that Level 2 was more critical on iRT spent on providing CA from upper levels. The Level 2 users are mostly satisfied with iRT from the lower level, while most of the L4 users are unsatisfied with iRT.

Table 1. Correlation matrix of responses.

\begin{tabular}{|c|c|c|c|c|c|c|c|c|c|}
\hline & & Q1 & Q2 & Q3 & Q4 & Q5 & Q6 & Q7 & Q8 \\
\hline Q1 & Quality solution from upper levels & 1.00 & & & & & & & \\
\hline Q3 & Quality solution from lower levels & -0.02 & -0.35 & 1.00 & & & & & \\
\hline Q4 & Time solution from lower levels & -0.06 & -0.18 & 0.66 & 1.00 & & & & \\
\hline Q6 & Quantity of case description field & -0.08 & -0.05 & 0.40 & 0.33 & 0.53 & 1.00 & & \\
\hline Q7 & Quality of additional questions & 0.44 & 0.32 & 0.40 & 0.33 & 0.37 & 0.12 & 1.00 & \\
\hline Q8 & Quantity of additional questions & 0.35 & 0.13 & 0.22 & 0.19 & 0.18 & -0.01 & 0.69 & 1.00 \\
\hline
\end{tabular}

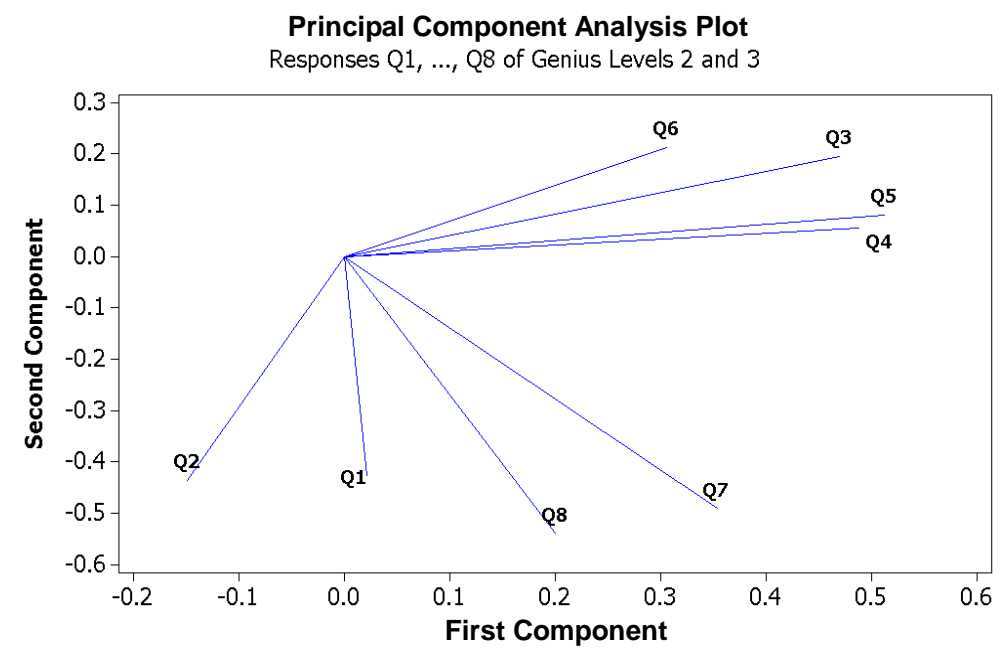

Figure 8. PCA plot of responses Q1, $\cdots, Q 8$ of genius levels 2 and 3 [7]. 

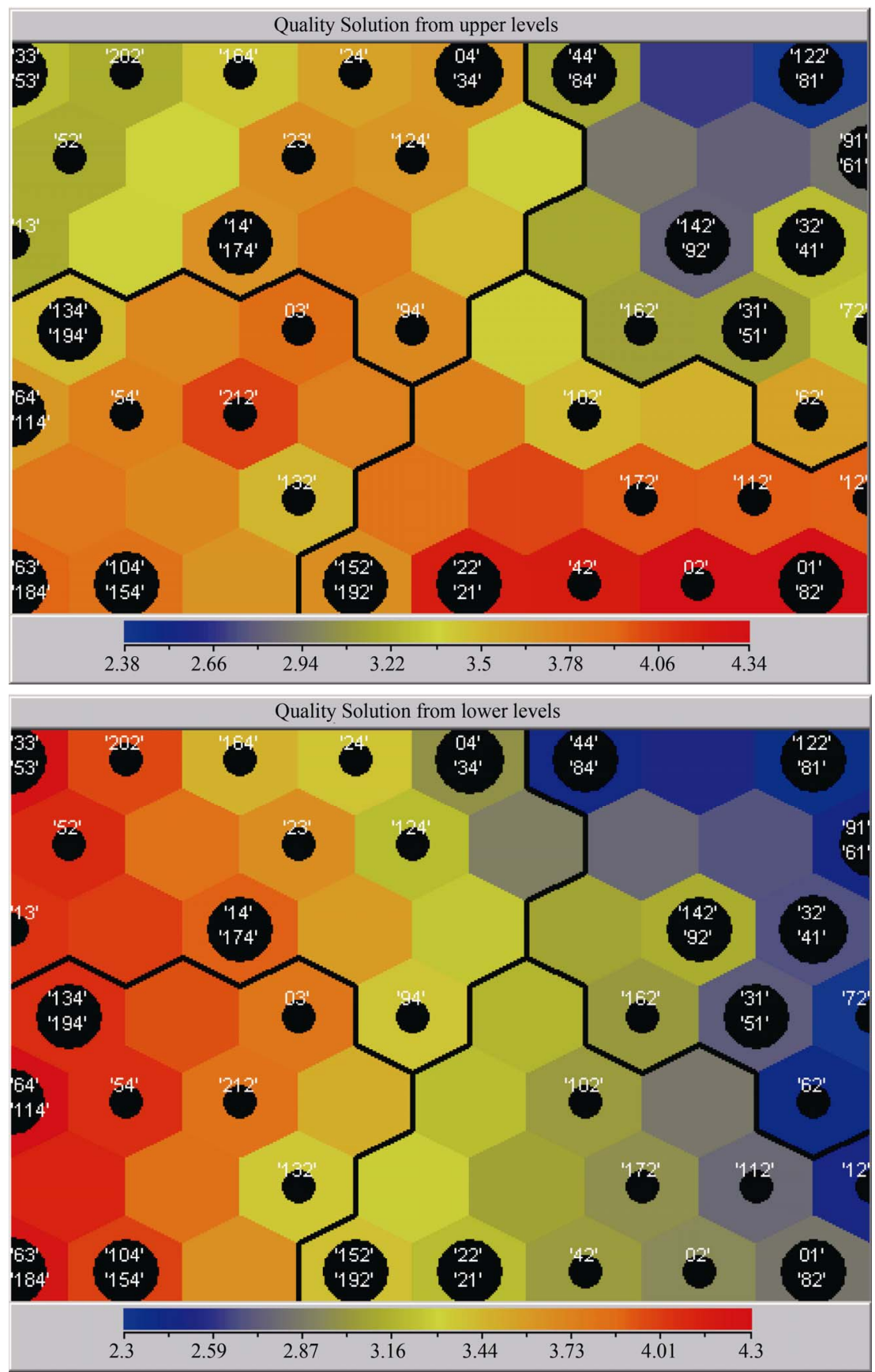

Figure 9. Quality of issue corrective actions (QoiCA) from upper and lower level.

\subsection{Issue Descriptions}

Having the description of the issues raised correctly in the first place helps to reduce the iRT for providing corrective actions.

From Figure 11, it can be seen that some of the L4 users are unsatisfied with the issue quality description provided from the lower levels in the chain. The quantity of the issue description can be too much, too little or just enough, so that the issues raised can be reproduced and resolved with good quality of CA at reasonable low iRT. The quantity field on the left of the figure reveals that some of the L4 users and a few L2 users are extremely unsatisfied.

\subsection{Additional Questions in CAP Genius Tool}

Figure 12 reveals the survey results concerning the additional questions requested by different levels. The tool provides fields for default additional questions such as 

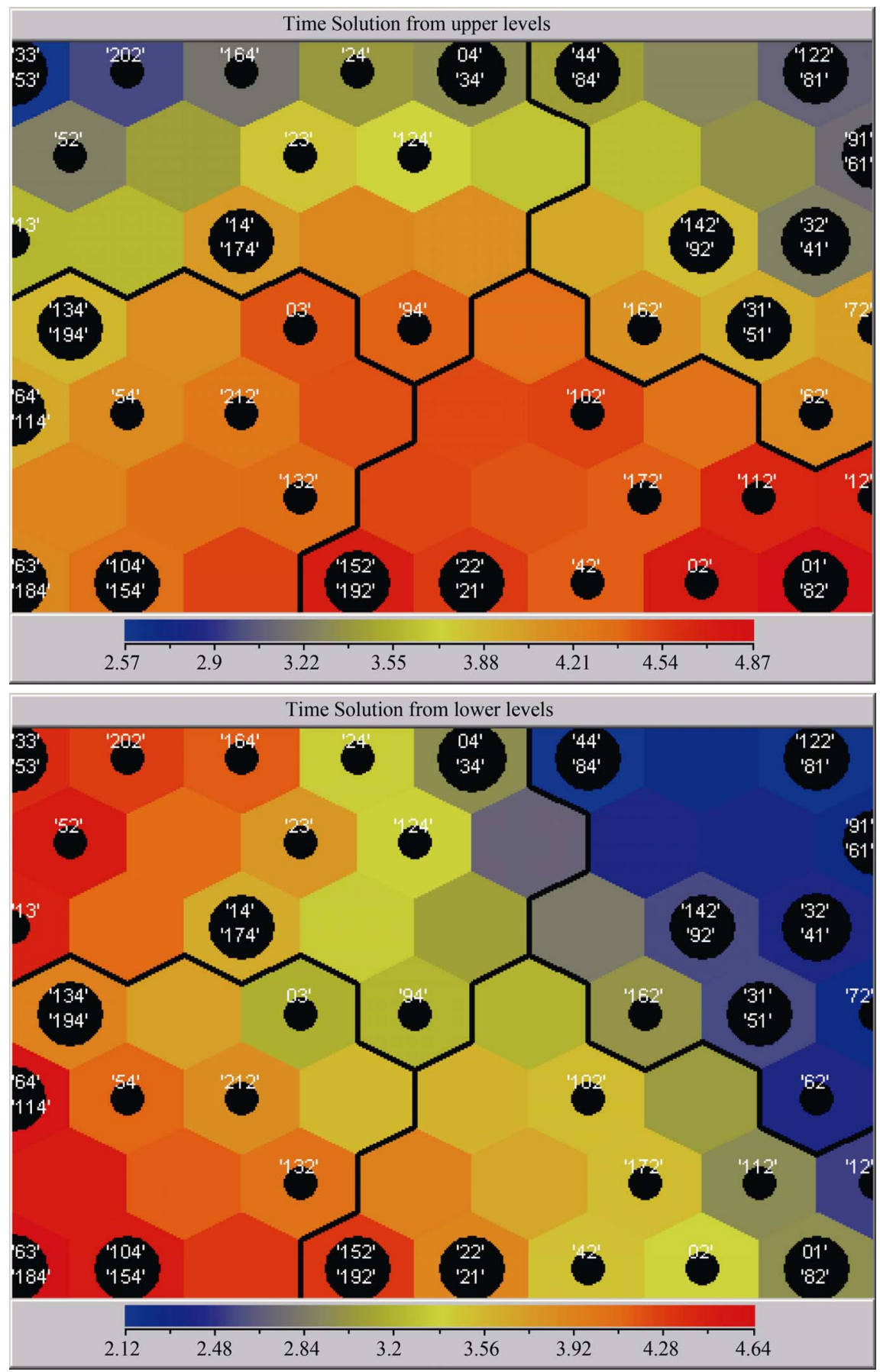

Figure 10. iRT from upper and lower levels.

software and hardware versions, severity of the issue etc. If additional information or questions are not provided or are not enough then a request for more information (see Figure 12) may be asked by issue resolvers, L2, L3 and L4, to clarify the issue reported in detail. L4 and L2 request more information from lower levels very often or often. Few of the level L1 and L2 users request more information from upper levels. The iRT to provide the
CA is increased whenever the issue resolvers have to request more information that was not provided in the additional questions. It can also be seen that with the exception of a few respondents, most of them were satisfied with the quality content of the additional questions. The quantities of the additional questions were averagely received well by most of the respondents from various levels. 

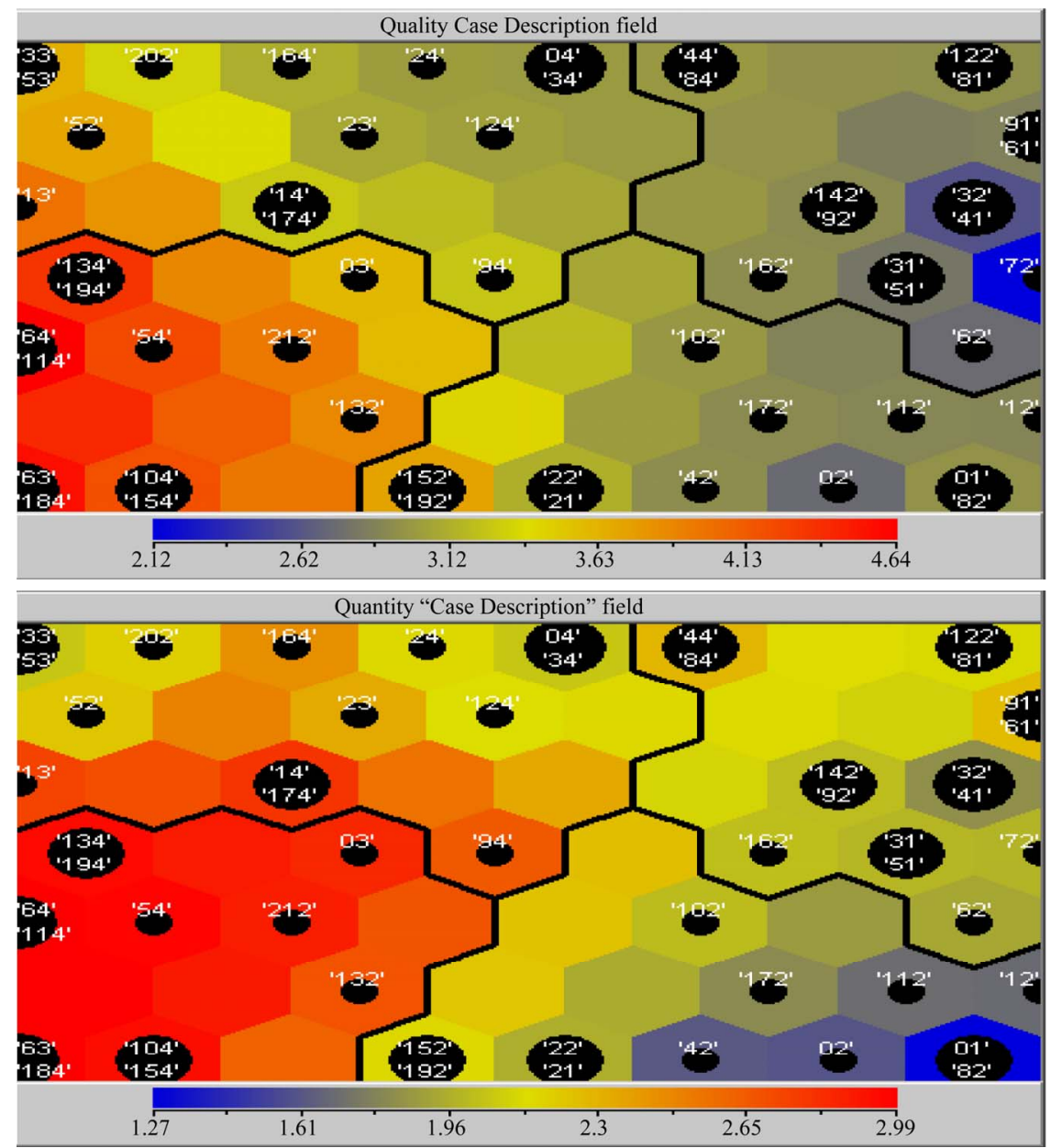

Figure 11. Quality and quantity of the issue description.

\section{Independent Variables}

The independent variables are the ones which are used to clarify some issues that need attention for improvement in the dependent variable discussed in the previous chapter.

\subsection{Business Justification}

Figure 13 illustrates how different service levels perceive the availability of the issue business impact provided the issues creator. Business justification helps to prioritize the importance of the issue. The more important the issue is, the higher the priority given. A large number of L4 users seem not to be satisfied with the business justification provided, while another considerable cluster of L2 users do seem to be satisfied. For a research and development group (R \& D), it's important to support a GENIUS issue with as much business case information as possible. This will lead to issues the highest cost being resolved faster, due to the business facts being clear to the BU management.

\subsection{Knowledge and Experience with Nokia's Products}

Figure 14 shows the experience, in months, and knowledge of participants on Nokia's products. Some of the L4 and L2 users have short experience but have high knowledge of Nokia's product. The Duration of working with Nokia products varied from 53 to 153 months.

\subsection{Genius Tool Training}

It is important that the people who are in the chain network for resolving issues from customers have a good knowledge of the tool used to escalate the customer's issue through the chain network i.e. from L1 to L4 and vice versa. Users of the GENIUS tool need to be trained to use it effectively. The following result illustrates how the participants have received the training for the corrective actions process (CAP) of the genius tool.

From Figure 15, it reveals that a small cluster of levels 1 and 2 (21, 22 and 61, 91, 41, 32, 31, 51 and 72) have not received enough training in using the tool. Duration 

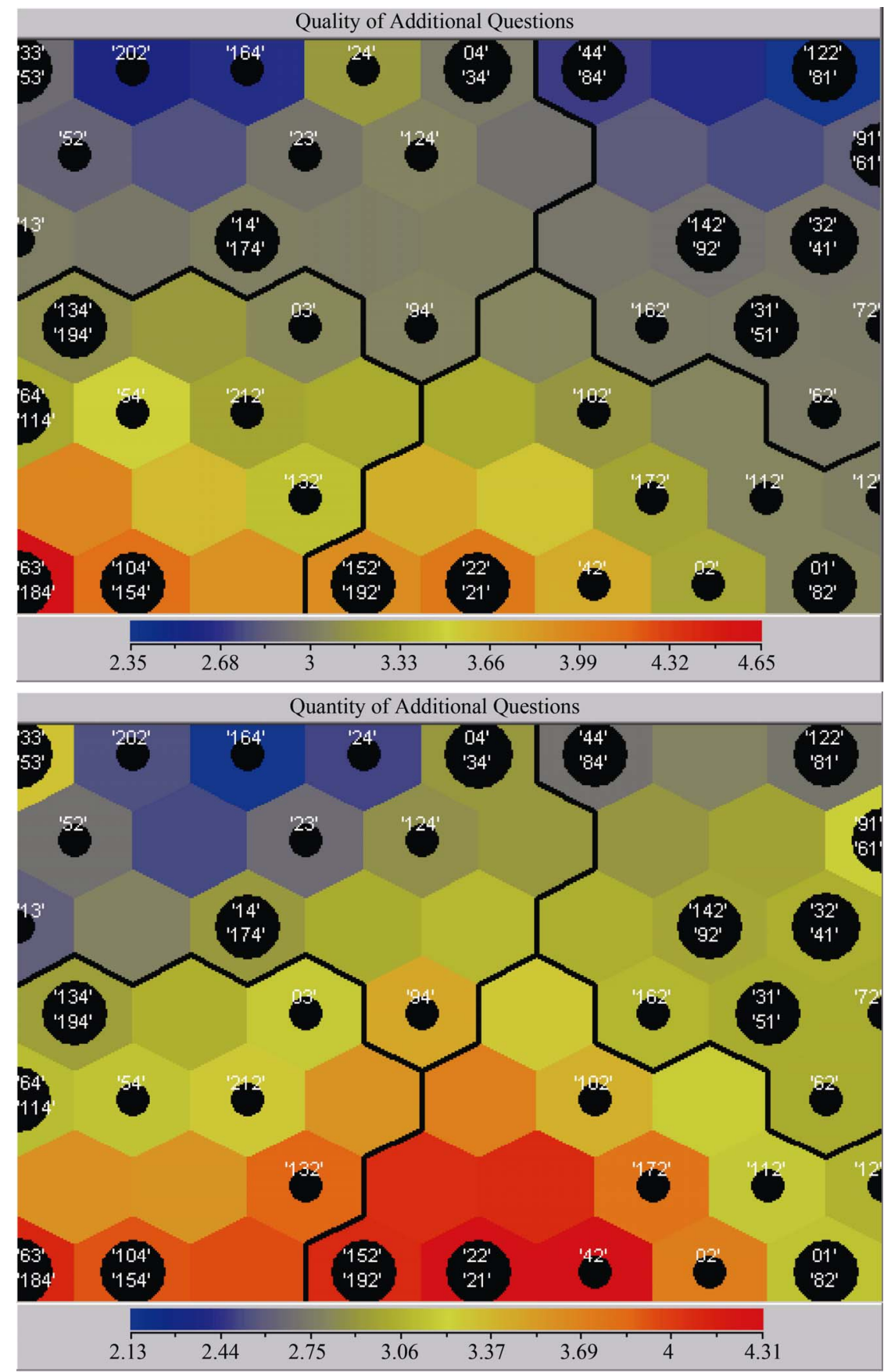

Figure 12. Quality and quantity of additional questions.

of GENIUS training was on average enough for the participants who received it. Some of the L1 and L2 users graded the training received to be poor as can be seen from the figure (01, 33, 52, 53, 72 and 82 ).

\subsection{Support, Satisfaction with Genius Tool}

Figure 16 demonstrates the perceived satisfaction of different service level on the use and support of the GENIUS tool. Key users of the GENIUS are contacted henever there is a technical usage problem with the tool. Key users are a part of the GENIUS training team. The above figure shows that most of the respondents know their GENIUS key users with the exception of a few in the L1 and L2 group (61, 91, 41, 32, 31 and 51). Some of the L1 and L2 users are clearly unsatisfied, while a few L4 users are very satisfied. A few clusters of L2 users need support for the GENIUS tool, while a few L4 users indicated that they were well trained in the daily usage of 


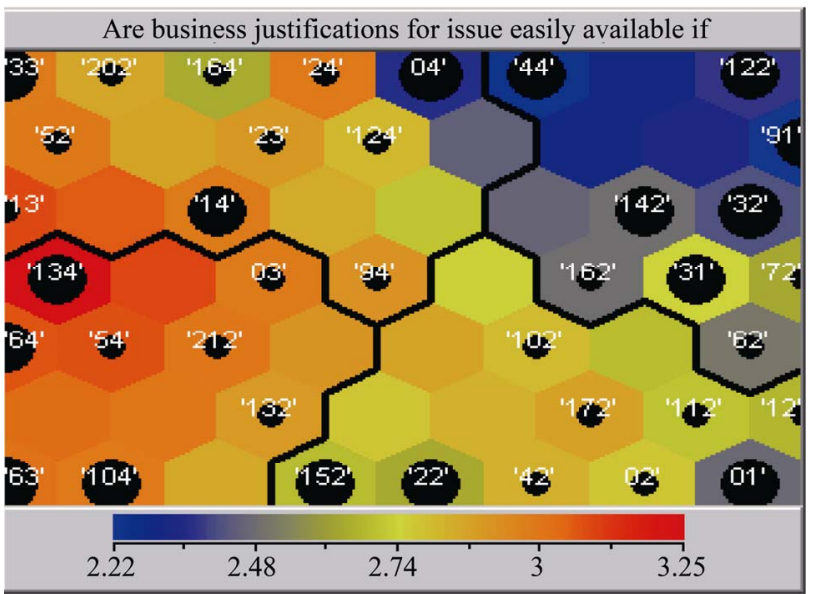

Figure 13. Business justification for issues presented by customers. the tool.

\subsection{Samples and More Information for Reproducing Issues}

Material e.g. samples of the terminals with a reported issue and supportive equipment are necessary for issue resolvers in verifying the issues by reproducing them. Getting samples for verifying an issue in time is crucial for achieving low iRT. Few L4 users experience a long time in receiving samples. In some cases, geographical factors contribute to extended times. In Figure 17 it can be seen that L2 as well L4 asks more information from lower levels.

\section{Conclusion}

Due to the nature of the data, clustering with The self-
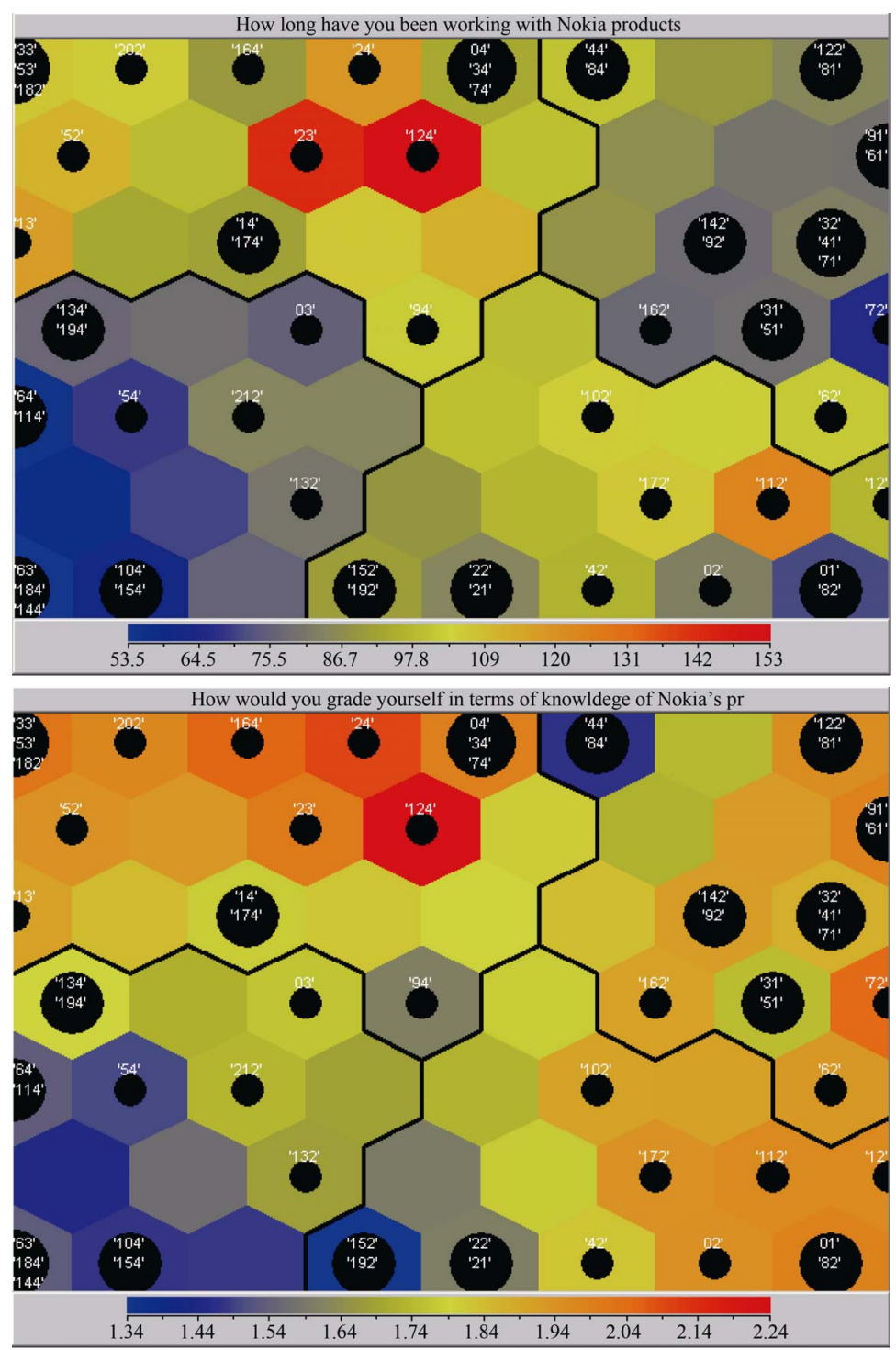

Figure 14. Experience and knowledge of Nokia's product. 


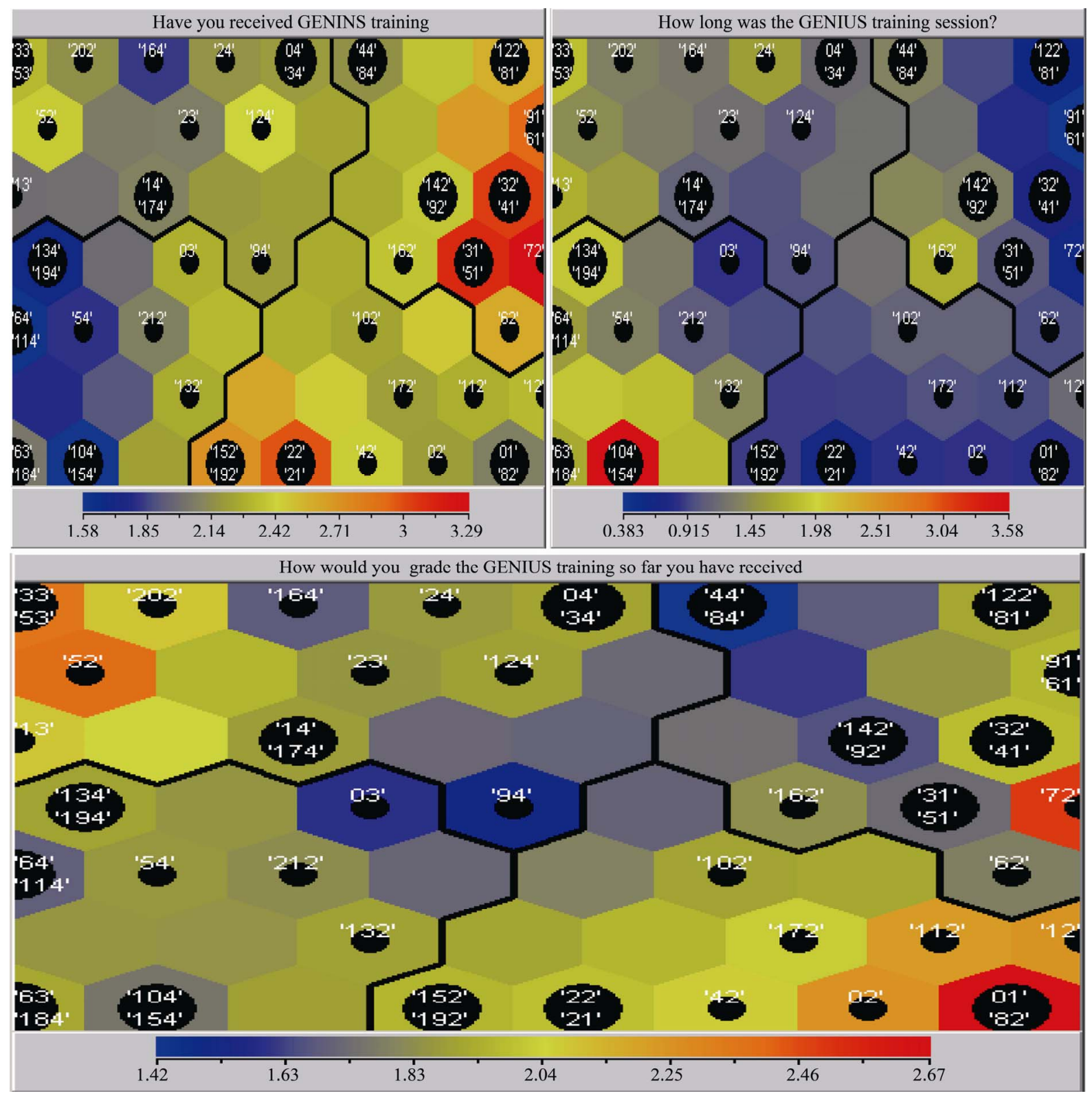

Figure 15. Genius training, duration and evaluation feedback.

organizing map approach was found to be the most applicable method for analyzing the survey data.The extreme ends of GENIUS user, i.e. L1 and L4, were not included in some dependent and independent variables because the tool does not go beyond L4 or below L1. However, in future work it would be interesting to go beyond the tool limits to explore a broader population and more findings. Some of the clear issues observed in this paper that contribute to long resolution time are (iRT): 1) Long time for receiving samples. This might, in some cases, be due to geographical factors and delays caused by customs; 2) A high frequency rate frequency of asking for more information from lower levels; 3)
Missing a business impact price tag, for enabling the resolvers to prioritize the QoiCA accordingly. It was also observed that quality of issue corrective actions (QoiCA) might be jeopardized by providing: 1) A poor description of the issue by the creator of the case; 2) A poor response to default additional question regarding the reported issue. L1 users need more training with the GENIUS tool so that they know, for example, where to get help when they need it. Future work will concentrate on the existing quality of the description of issues versus the QoiCA, iRT and communication between customer with issues and resolvers in order to increase the customer satisfaction and loyalty. 


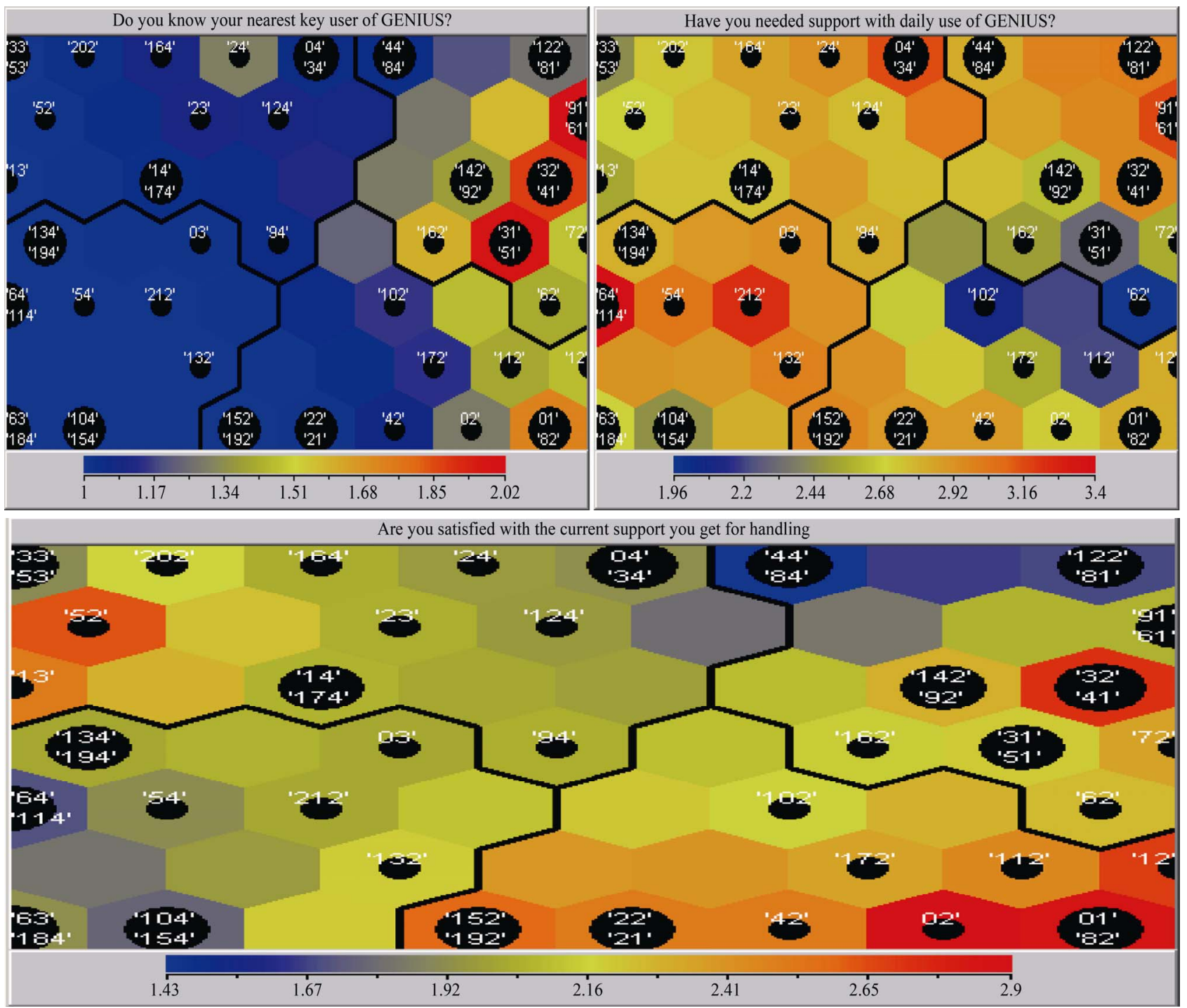

Figure 16. Key users, support and satisfaction using the genius tool for handling customer's issues.

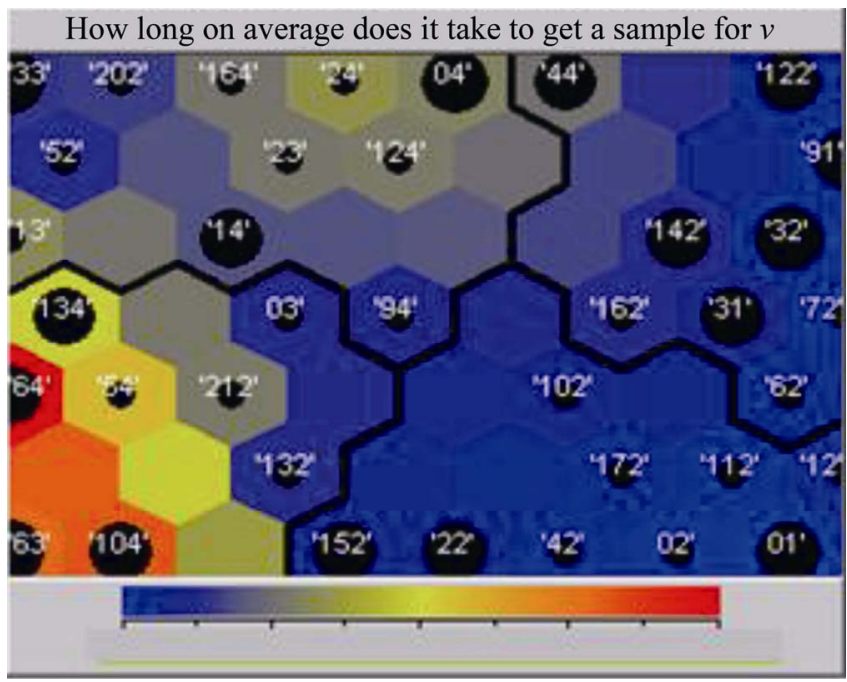



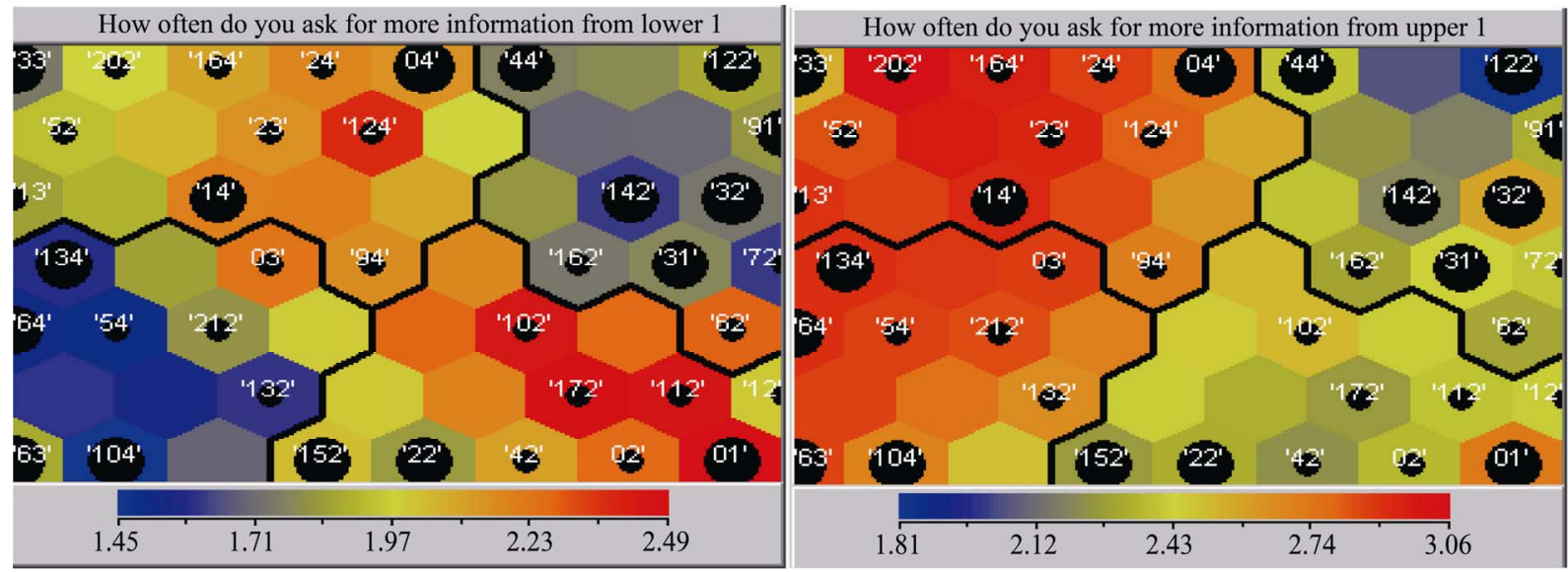

Figure 17. Samples and information request.

\section{Acknowledgements}

I would like to thank my work manager Mr. Ilkka Kuuluvainen for giving varied and valuable feedback to this work. Also, I would like to thank other work colleagues for language proof readings, especially David Powney, Brett Salahub and David Rios.

\section{REFERENCES}

[1] C. Lovelock, et al., "Services Marketing People, Technology, Strategy,” 6th Edition, Prentice Hall, Upper Saddle River, 2007.

[2] Technical Assistant Research Programs Institute (TARP), “Consumer Complaint in America,” 1995.

[3] B. Bergman and B. Klefsjo, "Quality: From Customer Needs to Customer Satisfaction,” McGraw Hill, London, 1994.

\section{Abbreviations and Acronyms}

ASV: Authorized Service Vendors.

BU: Business Unit.

CA: Corrective Actions.

CAP: Corrective Actions Process.

GENIUS: Global Exchange for Nokia Product Informa-
[4] M. G. Vega, et al., "Measuring Customer Satisfaction in a Service Company Combining Approaches for Quality Service Improvement,” Thesis at Växjö University, Sweden, 2003.

[5] T. Kohonen, "Self-Organizing Maps,” Springer Series in Information Sciences, Vol. 30, Springer, Berlin, Heidelberg, New York, 2001.

[6] A. Mwegerano, P. Kytösaho, et al., "Characterization of Resolution Cycle Times of Corrective Actions in Mobile Terminals," Journal of Quality and Reliability Engineering International, Vol. 24, No. 5, 2008, pp. 613-621.

[7] Hair, Anderson, Tatham and Black, "Multivariate Data Analysis,” 5th Edition, Prentice Hall, Upper Saddle River, 1998.

[8] M. Negnevitsky, “Artificial Intelligence Systems: A Guide to Intelligent Systems,” 3rd Edition, Pearson Education Limited, Harlow, 2001.

tion \& End User Support.

iRT: Issue Resolution Time.

L: Level of Service in the CAP GENIUS Chain Network.

SOM: Self-Organizing Maps.

R\&D: Research and Development.

QoiCA: Quality of Issue Corrective Actions. 